\title{
Benefits of Using a Biological Confocal Microscope to Resolve Difficult and Unusual Problems in Material Science
}

\author{
Earl J Sanford
}

Corning Incorporated, Characterization \& Materials Processing, Corning NY, USA

Most confocal microscopes designed specifically for looking at $\mathrm{m}$ aterial samples use reflected light to give you topographic infor mation about the sample' s surface. While these microscopes do a very good job at providing this info rmation, with resolutions down into the tens of nanom eters, there are some advantages of using a confocal microscope that is designed to image biological samples when examining certain materials. Biological confocal microscopes have the ability to capture fluorescent images and in some cases the ability to perform spectral analysis of the light that is returned to the microscope.

This talk will focus on how fluorescence c onfocal microscopy has been used to ex plore various issues with glass, ceramic, and polymeric samples. The ta lk will cover a broad range of sam ples and some basic principles of confocal microscopy - see Figure 1.

The fact that $\mathrm{m}$ any polymers and glass $\mathrm{f}$ laws will auto fluoresce makes the biological c onfocal microscope a good tool for studying these samples. If the sample will not fluoresce on its own, it may be possible to make the regions of interest fluoresce. It is also fairly easy to make fluorescent solutions that can be used to bring out textures in samples. We have determ ined that using flu orescence imaging generates better data sets of the pores and cracks in the ceramic than using reflected light imaging. When this method is used, either an oil or a wax with a dye in it are embedded into the sample - see Figure 2.

Some of the samples to be discussed during the talk include:

- examination of coatings applied to polymer beads used as micro-carriers

- measurement of polymer film thickness

- studying dynamic events such as acid attack of glass surfaces

- using the fluorescence capabilities to study textures and features within glass, ceramics and polymers

- $\quad$ using the spectral analyzer to look at photoluminescence

A few example of using a fluorescence confocal microscope with reflected light will also be presented just to show that these measurements can be made as well.

The examples that are being pres ented in th is talk have com e from several different brands of microscopes and are not aimed at promoting any one brand over another. The main selection criteria for the microscope should be its capab ilities - i.e. objectives and lase rs available. And obviously, the sample to be examined must also fit on the microscope.

References:

1. J. B. Pawley, "Handbook of Biological Confocal Microscopy", Third Edition, Springer, New York, 2006 


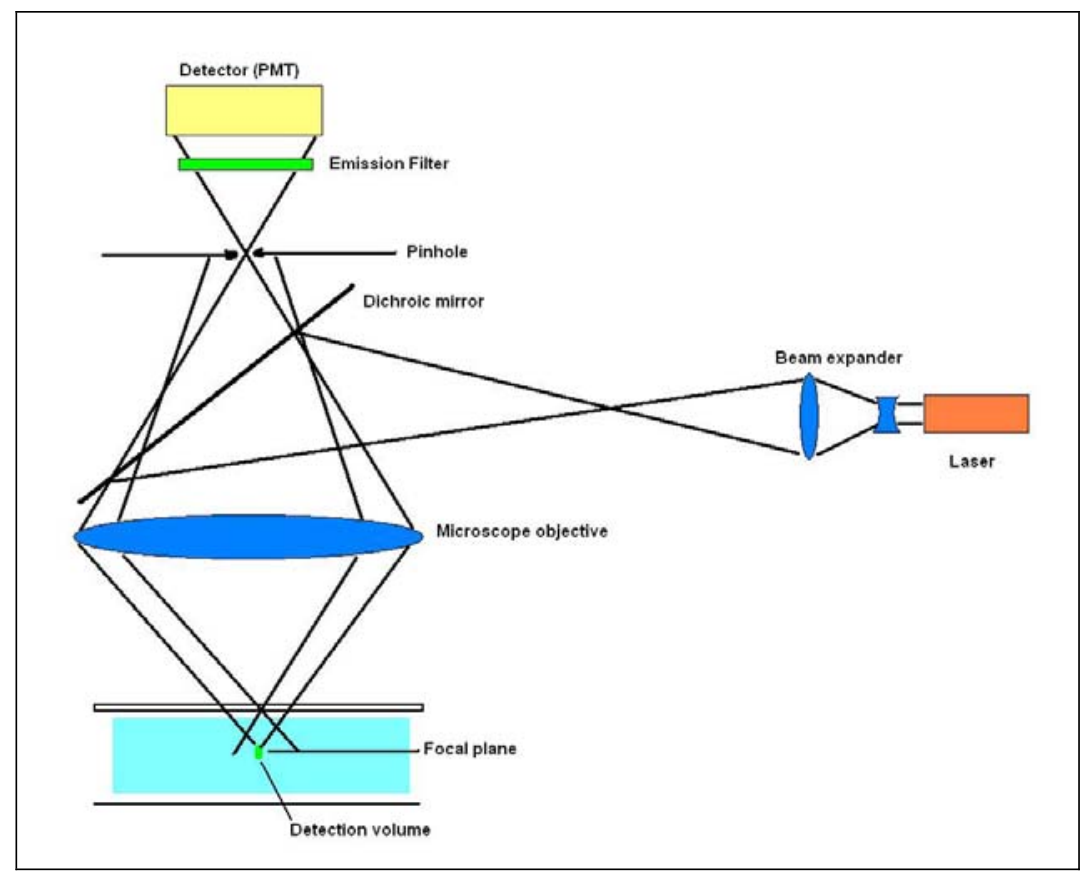

Figure 1 - How a confocal $\mathrm{m}$ icroscope works. Laser scanning confocal microscopes work by scanning one or more laser points over a sample and then co llects the signals of interest back and builds up an image of the sample point by point. The light that is collected from the sample passes through a variable aperture, called a pinhole, that is reduced to a very small size to induce confocality - see Figure 1 . The main impact of this pinhole is the removal of out-of-focus light com ing from the sample. This results in an image that has high contrast and turns the microscope into a depth-discriminating optical system. It is therefore possible with a confocal m icroscope to exclusively image a thin "optical slice" out of a thick section, a method known as optical sect ioning. Collecting a series of thes e of these optical slices, called a z-stack, allows for the measurement and display of 3D information

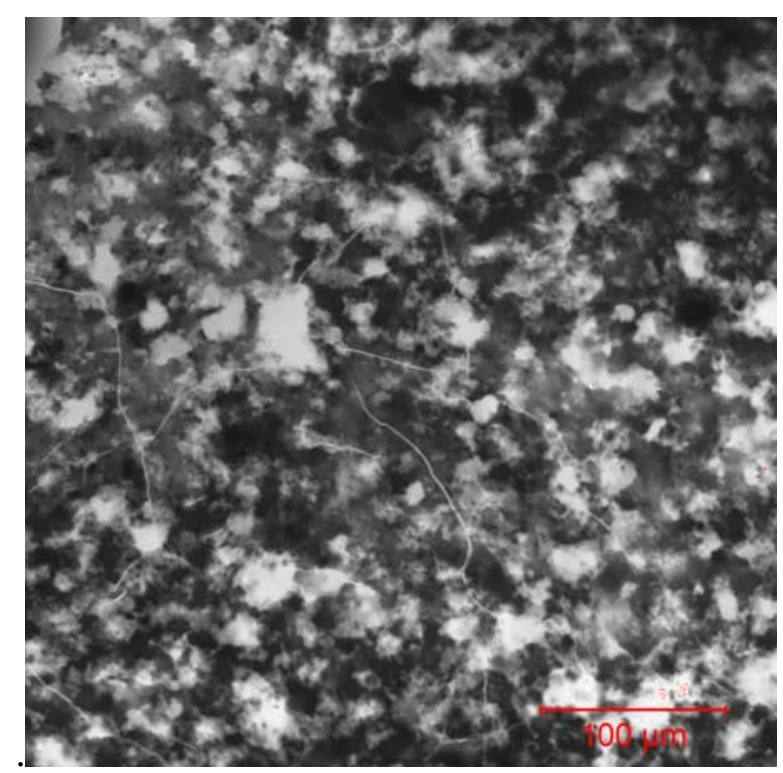

Figure 2 - A confocal image that shows numerous pores and cracks (bright regions) in a piece of ceramic material. The pores and cracks are filled with a fluorescent solution. 